\title{
MicroRNA levels in bone and blood change during bisphosphonate and teriparatide therapy in an animal model of postmenopausal osteoporosis
}

Roland Kocijan $^{1,2}$, Moritz Weigl ${ }^{3}$, Susanna Skalicky ${ }^{3}$, Elisabeth Geiger $^{3}$, James Ferguson ${ }^{1,4}$, Gabriele Leinfellner $^{1,4}$, Patrick Heimel ${ }^{1,4,5}$, Peter Pietschmann ${ }^{6}$, Johannes Grillari ${ }^{4,7}$, Heinz Red1 ${ }^{1,4}$, Matthias Hackl $^{3,4}$

1. Ludwig Boltzmann Institute for Experimental and Clinical Traumatology in AUVA research center; Donaueschingenstraße 13, 1200 Vienna, Austria

2. Hanusch Hospital, 1st Medical Department, Heinrich Collin-Str. 30, 1140 Vienna, Austria

3. TAmiRNA GmbH, Muthgasse 18, 1190 Vienna, Austria

4. Austrian Cluster for Tissue Regeneration, Austria

5. Karl Donath Laboratory for Hard Tissue and Biomaterial Research, Department of Oral Surgery, Medical University of Vienna, Austria

6. Department of Pathophysiology and Allergy Research, Center for Pathophysiology, Infectiology and Immunology, Medical University of Vienna, Vienna, Austria.

7. Christian Doppler Laboratory on Biotechnology of Skin Aging, Department of Biotechnology, BOKU - University of Natural Resources and Life Sciences Vienna, Vienna, Austria.

Abbreviated title: MicroRNAs and Microstructure in Osteoporosis

Corresponding author and person to whom reprint requests should be addressed:

Dr. Matthias Hackl

TAmiRNA GmbH

Muthgasse 18

1190 Vienna, Austria

matthias.hackl@tamirna.com

+431476546587 


\section{Disclosure statement:}

Roland Kocijan, Elisabeth Geiger, James Ferguson, Gabriele Leinfellner, Patrick Heimel and Peter Pietschmann state that they have no conflicts of interest. Matthias Hackl and Johannes Grillari are co-founders of TAmiRNA. Matthias Hackl, Susanna Skalicky and Moritz Weigl are employed by TAmiRNA GmbH. Johannes Grillari and Heinz Redl are scientific advisors to TAmiRNA. Matthias Hackl and Johannes Grillari hold patents related to the use of circulating microRNAs as biomarkers for bone diseases.

\section{Funding:}

This study was supported by the FFG Feasibility Project Grant 852770, the Christian Doppler Gesellschaft, EU-FP7 Health Project FRAILOMIC 305483 and EU-FP7 Health Project SYBIL 602300. 


\section{ABSTRACT (316 words)}

MicroRNAs control the activity of a variety of genes that are pivotal to bone metabolism. Therefore, the clinical utility of miRNAs as biomarkers and drug targets for bone diseases certainly merits further investigation. This study describes the use of an animal model of postmenopausal osteoporosis to generate a comprehensive dataset on miRNA regulation in bone tissue and peripheral blood during bone loss and specifically anti-resorptive and osteoanabolic treatment.

Forty-two Sprague-Dawley rats were randomized to SHAM surgery $(\mathrm{n}=10)$ or ovariectomy $(\mathrm{OVX}, \mathrm{n}=32)$. Eight weeks after surgery, OVX animals were further randomized to antiresorptive treatment with zoledronate $(n=11)$, osteo-anabolic treatment with teriparatide $(n=11)$, or vehicle treatment $(n=10)$. After 12 weeks of treatment, bone and serum samples were used for microRNA analysis using next-generation sequencing (NGS), mRNA levels using RT-qPCR, and bone microarchitecture analysis using nanoCT.

Ovariectomy resulted in loss of trabecular bone, which was fully rescued using osteo-anabolic treatment, and partially rescued using anti-resorptive treatment. NGS revealed that both, antiresorptive and anabolic treatment had a significant impact on miRNA levels in bone tissue and serum: out of 426 detected miRNAs, 46 miRNAs were regulated by teriparatide treatment an $\mathrm{d} 10$ by zoledronate treatment (p-adj. < 0.1). Interestingly, teriparatide and zoledronate treatment were able to revert miRNA changes in tissue and serum of untreated OVX animals, such as the up-regulation of miR-203a-3p, a known osteo-inhibitory miRNA. We confirmed previously established mechanisms of miR-203a by analyzing its direct target Dlx 5 in femoral head.

Our data reveal a significant effect of ovariectomy-induced bone loss, as well as the two major types of anti-osteoporotic treatment on miRNA transcription in femoral head tissue. These changes are associated with altered activity of target genes relevant to bone formation, such as Dlx5. The observed effects of bone loss and treatment response on miRNA levels in 
bone are also reflected in the peripheral blood, suggesting the possibility of minimallyinvasive monitoring of bone-derived miRNAs using liquid biopsies.

\section{Highlights:}

1. microRNA expression in bone tissue is altered by osteo-anabolic and anti-resorptive therapy in OVX rats.

2. microRNA changes in untreated OVX rats are reverted by anti-osteoporotic therapy.

3. miR-203a is up-regulated during bone loss and down-regulated following therapy.

4. Bone tissue and serum levels of miR-203a are highly correlated.

Key Words: bone microstructure, circulating microRNA, osteoporosis, bisphosphonate, parathyroid hormone (PTH), $\mu \mathrm{CT}$, RUNX2

\section{INTRODUCTION}

Osteoporosis is a worldwide affliction associated with low-traumatic fractures, morbidity and mortality ${ }^{(1-3)}$. Currently available biomarkers for the diagnosis and follow-up of bone diseases are bone turnover markers $(\mathrm{BTMs}){ }^{(4)}$. BTMs reflect bone resorption and bone formation and therefore the activity of bone cells, and are recommended for monitoring of treatment response ${ }^{(5)}$. MicroRNAs (miRNAs) are a novel group of biomarkers ${ }^{(6)}$. miRNAs are small non-coding RNAs that are involved in several biological processes such as stem cell differentiation, cell cycle control, apoptosis, aging and bone metabolism ${ }^{(7-10)}$. In regards to bone metabolism and bone health, several miRNAs have been identified as potent regulators of osteogenic differentiation and osteoclast formation ${ }^{(11)}$. Interestingly, genetic association studies have also identified polymorphisms in miRNA binding sites in 3'UTRs of FGF2, ON, 
and COL1A2 to be associated with bone mineral density ${ }^{(12)}$. Vice-versa, individuals carrying WNT1 mutations and thus exhibiting severe bone loss and multiple fractures exhibit particular miRNA profiles in their serum ${ }^{(13)}$, including down-regulation of miR-31-5p, a putative regulator of WNT1. In addition, miR-31-5p was shown to inhibit bone formation via RUNX2 and SATB2 ${ }^{(14)}$, while inhibition of miR-31-5p activity using antisense RNAs was shown to improve fracture healing in vivo ${ }^{(15)}$. Finally, using ovariectomized mice, changes in expression of miRNAs and mRNAs in femur and tibia were identified already after a period of 4 weeks, and found to be associated with reduced bone formation ${ }^{(16)}$.

Hence, circulating miRNAs have been proposed as potentially valuable biomarkers for the diagnosis of osteoporosis and the prediction of low-traumatic fractures ${ }^{(6)}$, based on studies performed in women with postmenopausal osteoporosis with and without low-traumatic fractures ${ }^{(17-19)}$, and the observation that circulating miRNAs are associated to bone microstructure and histomorphometry (20).

Although we recently observed in a cross-sectional study that previous anti-resorptive therapy did not significantly alter serum miRNA levels in osteoporotic individuals, the effect sizes observed for treated versus untreated subjects suggested a potential response of miRNA biomarkers to therapeutic intervention (21). In addition, a recent study performing targeted analysis of a small set of circulating miRNAs in patients undergoing denosumab or teriparatide treatment revealed several significant changes ${ }^{(22) . ~ W h i l e ~ b i s p h o s p h o n a t e s ~ s t i l l ~}$ represent the most commonly prescribed type of osteoporosis drug, there are no data describing drug effects on miRNA levels in bone tissue and circulation. Nevertheless, such data are important to validate the clinical utility of novel bone biomarkers for diagnosis and monitoring response to treatment in bone diseases and could potentially unravel novel drug targets for intervention. Accordingly, we designed an animal trial that would enable us to systematically analyze miRNA levels in bone tissue and serum during bone loss and 
therapeutic intervention. We specifically considered the analysis of common changes in bonerelated miRNAs at tissue and cell-free blood level (serum) after a 12-week treatment course, as well as time-dependent changes of bone-related miRNAs in serum including associations to bone microstructure under anti-resorptive and osteo-anabolic therapy, respectively.

Given the large number of samples and data generated, the primary objective of the present study was to evaluate the expression of bone-related miRNAs in bone and serum under parathyroid hormone (teriparatide) or zoledronic acid (zoledronate) treatment in an OVXmodel for postmenopausal osteoporosis after a full course of treatment. We hypothesize that: (i) ovariectomy induced bone-loss will result in specific changes of bone-related miRNAs compared to SHAM controls, (ii) that both zoledronic acid and teriparatide treatment might rescue these specific changes, and (iii) that the levels of these miRNA signatures in peripheral blood are correlated to bone tissue levels based on their release from bone cells.

\section{MATERIALS \& METHODS}

This study was conducted at the Ludwig Boltzmann Institute for Traumatology, Vienna, Austria in cooperation with TAmiRNA GmbH (Vienna, Austria). The present study is part of

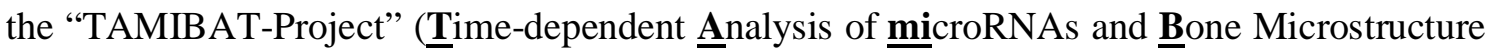

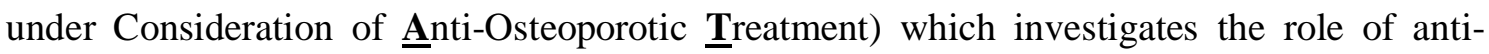
osteoporotic treatment on bone-related miRNAs in bone tissue and serum.

\subsection{Experiment Design}

In total, 42 Sprague Dawley rats were either ovariectomized (OVX) or sham-operated at the age of 6 months. Eight weeks following OVX treatment, 32 animals were randomly assigned into three groups: (i) OVX + vehicle (VEH), n= 10; (ii) OVX + teriparatide (TPD), n=11; or 
(iii) OVX + zoledronic acid (ZOL), n=11. A fourth group was (iv) sham-operated (SHAM), $\mathrm{n}=10$, and received placebo parallel to the OVX groups. Animals in group (i) received zoledronic acid (100 $\mu \mathrm{g} / \mathrm{kg} /$ body weight s.c., single dose, Novartis Pharma AG). Animals in group (ii) received teriparatide s.c. periodically (weekly dose $210 \mu \mathrm{g} / \mathrm{kg} / \mathrm{body}$ weight (= 42 $\mu \mathrm{g} / \mathrm{kg} /$ body weight/per day over 5 days), Eli Lilly and Company). Group (iii) and (iv) acted as controls and received vehicle injections $\left(0.9 \%\right.$ saline with $20 \mathrm{mM} \mathrm{NaH}_{2} \mathrm{PO}_{4}$ in $0.9 \% \mathrm{NaCl}, 3$ $\mathrm{mg} / \mathrm{ml}$ mannitol - dosed at a volume of $1 \mathrm{ml} / \mathrm{kg}$ body weight) s.c. at the same interval as group (ii).

\subsection{Blood Collection}

Blood was taken at the tail vein at week $0,2,8,10,12,16$ and 20. Blood sampling was performed in the early afternoon after a 6-hour fasting period to avoid influences of nutrition on bone metabolism. Blood serum was sampled in Eppendorf tubes from the lateral tail vein under short inhalation anesthesia (isoflurane $1-3 \%$, oxygen). Clotting time for the serum samples was $30-45$ minutes at room temperature. Samples were then centrifuged at $2500 \mathrm{xg}$ for 15 minutes at $20^{\circ} \mathrm{C}$. The same inhalation anesthesia protocol was used during in vivo micro-CT measurements. Study duration was 20 weeks for all subgroups, of which the treatment period accounted for 12 weeks. The complete study design is shown in Figure 1A.

\subsection{Animals}

Thirty-two ovariectomized and 10 sham-operated 6-month-old female Sprague Dawley rats (ranging in weight from $270 \mathrm{~g}$ to $356 \mathrm{~g}$ ) were sourced from Charles River Laboratories, Germany for this study. Animals were kept in makrolon type 4 cages with two animals per cage. They were fed ready-made feed (Rat Maintenance, Ssniff GmbH) and water ad libitum. Bedding was obtained from Abedd GmbH (Midi Chips). Gnawing blocks and tunnels were provided as cage enrichment. A sample size calculation was carried out based on primary 
variable bone density. Effect sizes from previous studies ${ }^{(23,24)}$ were used in the calculation. A difference in the group average of $15 \%$ and a power of $90 \%$ was assumed, a group size of 10 was, therefore, adequate. Analogous studies have reported similar group sizes (23,24). All experimental protocols were approved in advance by the Municipal Government of Vienna in accordance with Austrian law and the Guide for the Care and Use of Laboratory Animals as defined by the National Institute of Health (revised 2011).

\subsection{MicroRNA Analysis Serum}

\subsubsection{RNA Extraction}

Total RNA was extracted from $200 \mu \mathrm{l}$ serum using the miRNeasy Mini Kit (Qiagen, Germany). Samples were thawed on ice and centrifuged at 12,000 x g for 5 minutes to remove any cellular debris. For each sample, $200 \mu \mathrm{L}$ of serum were mixed with $1000 \mu \mathrm{L}$ Qiazol and 1 $\mu \mathrm{L}$ of a mix of 3 synthetic spike-in controls (Exiqon, Denmark). After a 10-minute incubation at room temperature, $200 \mu \mathrm{L}$ chloroform were added to the lysates followed by cooled centrifugation at $12,000 \mathrm{x}$ g for 15 minutes at $4^{\circ} \mathrm{C}$. Precisely $650 \mu \mathrm{L}$ of the upper aqueous phase were mixed with $7 \mu \mathrm{L}$ glycogen $(50 \mathrm{mg} / \mathrm{mL})$ to enhance precipitation. Samples were transferred to a miRNeasy mini column where RNA was precipitated with $750 \mu \mathrm{L}$ ethanol followed by automated washing with RPE and RWT buffer in a QiaCube liquid handling robot. Finally, total RNA was eluted in $30 \mu \mathrm{L}$ nuclease free water and stored at $-80^{\circ} \mathrm{C}$ to await further analysis.

2.4.2. MicroRNA reverse-transcription quantitative PCR Analysis in serum RNA (RT$q P C R)$

Starting from total RNA samples, cDNA was synthesized using the Universal cDNA Synthesis Kit II (Exiqon, Denmark). Reaction conditions were set in accordance to the manufacturer's specifications. In total, $2 \mu \mathrm{L}$ of total RNA were used per $10 \mu \mathrm{l}$ reverse 
transcription (RT) reaction. To monitor RT efficiency and presence of impurities with inhibitory activity, a synthetic RNA spike-in (cel-miR-39-3p) was added to the RT reaction. PCR amplification was performed in a 96-well plate format in a Roche LC480 II instrument (Roche, Germany) using EXiLENT SYBR Green mastermix (Exiqon, Denmark) with the following settings: $95^{\circ} \mathrm{C}$ for $10 \mathrm{~min}, 45$ cycles of $95^{\circ} \mathrm{C}$ for $10 \mathrm{~s}$ and $60^{\circ} \mathrm{C}$ for $60 \mathrm{~s}$, followed by melting curve analysis. To calculate the cycle of quantification values (Cq-values), the second derivative method was used. Spike-in control values were used for monitoring data quality (Supporting Figure 1). Cq-values of endogenous miRNAs were normalized to the RNA spike-in controls by subtracting the individual miRNA Cq-value from the RNA SpikeIn $\mathrm{Cq}$, thus obtaining delta-Cq $(\mathrm{dCq})$ values that were used for the analysis. Hemolysis was assessed in all samples using the ratio of miR-23a-3p versus miR-451a and applying a cut-off of a delta $\mathrm{Cq}>7$ indicates a high risk of hemolysis (25)

\subsection{MicroRNA/mRNA Analysis in Bone Tissue}

\subsubsection{RNA Extraction}

Femur bones were harvested from all rats. Any attached tissue was quickly removed and samples were immediately snap frozen in liquid nitrogen and stored at $-80^{\circ} \mathrm{C}$. For RNA extraction, only femoral heads of femur bones were used. For tissue homogenization, frozen bones were again put into liquid nitrogen and each bone was subsequently taken out and fixed in a jaw vice. Femoral heads were rapidly dissected at the femoral neck using a gigli wire saw, weighed and put back into liquid nitrogen. Femoral head samples were homogenized as described by Carter et al. (26). Each bone sample was added to a MPBio tube prechilled in liquid nitrogen containing Lysing Matrix M (MPBiomedicals, USA) followed by addition of $1000 \mu \mathrm{l}$ Qiazol. Tubes containing the bone sample, Lysing Matrix M and $1000 \mu \mathrm{l}$ Qiazol were put back in liquid nitrogen. Bone samples were then lysed by bead disruption in a MPBio Fastprep 24 instrument (MPBiomedicals, USA). After lysis, the tubes were centrifuged at 
$8600 \mathrm{x} \mathrm{g}$ for 30 seconds and the resulting supernatant was used for total RNA extraction using the miRNeasy Mini kit (Qiagen, Germany) as per the manufacturer's recommendation.

\subsubsection{Small RNA Sequencing}

Equal amounts of total RNA (100 ng) were used for small RNA library preparation using the CleanTag small RNA library preparation kit (TriLink Biotechnologies). Adapter-ligated libraries were reverse transcribed and amplified (19 cycles) using barcoded Illumina reverse primers in combination with the Illumina forward primer according to the instructions of the manufacturer. In total 15 small RNA sequencing libraries were prepared from bone tissue RNA extracts and used for equimolar pooling based on DNA-1000 high-sensitivity bioanalyzer results (Agilent Technologies). The library pool was sequenced on a NextSeq 500 sequencing instrument (50 bp cycles) following the manufacturer's instructions. Raw data was de-multiplexed and FASTQ files for each sample was generated using the bcl2fastq software (Illumina inc.). FASTQ data were checked using the FastQC tool (http://www.bioinformatics.babraham.ac.uk/projects/fastqc/). Sequencing reads were adapter trimmed and filtered for low-quality reads $(\mathrm{Q}<30)$. MicroRNA annotation was performed on the basis of sequential alignments against the rat genome reference and miRBase release 21 . Read counts were normalized to the total number of reads detected per sample to obtain the "tags per million" (TPM) for each miRNA and sample. Exploratory data analysis was performed using Clustvis (https://biit.cs.ut.ee/clustvis/) ${ }^{(27)}$.

\subsubsection{MessengerRNA (mRNA) reverse-transcription quantitative PCR Analysis (RT- $q P C R)$}

For mRNA quantification the TATAA SYBR Grandmaster mix kit (TATAA Biocenter, Sweden) was used. $25 \mathrm{ng}$ of bone tissue total RNA extracts were used for reverse transcription and all steps were carried out according to recommendations by the 
manufacturer. PCR amplification was performed in a 96 well format in a Roche LC480 II instrument (Roche, Germany) with the following settings: $95{ }^{\circ} \mathrm{C}$ for 30 seconds followed by 45 cycles of $95{ }^{\circ} \mathrm{C}$ for 5 seconds, $63{ }^{\circ} \mathrm{C}$ for 15 seconds and $72{ }^{\circ} \mathrm{C}$ for 10 seconds and subsequent melting curve analysis. To calculate the cycle of quantification values (Cqvalues), the second derivative method was used.

Table 1: Primer sequences used for mRNA reverse-transcription quantitative PCR analysis.

\begin{tabular}{|l|l|l|}
\hline Gene & Forward Primer & Reverse Primer \\
\hline GAPDH & AGACAGCCGCATCTTCTTGT & CTTGCCGTGGGTAGAGTCAT \\
\hline DLX5 & CTCTCTAGGACTGACGCAAAC & GAGTTACACGCCATAGGGTC \\
\hline RUNX2 & CAGACCAGCAGCACTCCATA & GCTTCCATCAGCGTCAACAC \\
\hline OSX & CGGCAAGGCTTCGCATCTG & GGAGCAGAGCAGACAGGTGAACT \\
\hline
\end{tabular}

2.5.4. MicroRNA reverse-transcription quantitative PCR Analysis in bone RNA (RT$q P C R)$

Bone microRNA analysis was performed similarly to serum microRNA analysis with the following modifications: precisely $10 \mathrm{ng}$ of total RNA were used for reverse transcription. Variance in reverse transcription was analyzed using spike-in controls (cel-miR-39). To account for variance in reverse transcription, qPCR data were normalized to cel-miR-39 (delta-Cq method).

\subsection{Bone Microstructure Assessment}

Micro-CT-scans were performed at the vertebral body L4 and the tibia in all animals. In-vivo $\mu \mathrm{CT}$ scans were performed using a SCANCO vivaCT 75 (SCANCO Medical AG, Brüttisellen, Switzerland) at week 0 (baseline), 8 and 12. Scans were performed at $70 \mathrm{kVp}$, 
$114 \mu \mathrm{A}, 500$ projections $/ 180^{\circ}$ with an integration time of $300 \mathrm{~ms}$ and reconstructed to an isotropic resolution of $45 \mu \mathrm{m}$.

The bone microstructure at study end (week 20) was evaluated using a $\mu \mathrm{CT}$ imaging system (nano-CT, $\mu$ CT 50, SCANCO Medical AG, Brüttisellen, Switzerland). The tube was operated at $90 \mathrm{kVp}, 200 \mu \mathrm{A}, 1000$ projections $/ 180^{\circ}$ with an integration time of $500 \mathrm{~ms}$. The scans were performed at an isotropic nominal resolution of $10 \mu \mathrm{m}$. The long axis of the biopsy specimen was oriented along the rotation axis of the scanner.

Image segmentation was performed using Definiens Developer XD 2.1.1 (Definiens AG, Munich, Germany). The tibia scans were downsized to $12.5 \%$ and maintained at $375 \mathrm{mg}$ $\mathrm{HA} / \mathrm{cm}^{3}$. Void volume inside and outside of the bone was separated by coating the bone tissue 2 voxel $(160 \mu \mathrm{m})$ thick with a temporary class. Void volume in contact with the image border was classified as exterior void and void enclosed by bone was classified as interior void. The temporary coating was removed by growing the void classes into the temporary class. The fibula was excluded from further measurements. As the cross-section area of the fibula was categorically smaller than the cross-section area of the rest of the tibia, it could be automatically distinguished and excluded. Cortical and trabecular bone was distinguished using a series of surface tension constrained region growing steps. The exterior void was coated as cortical bone into bone constrained by surface tension. This resulted in a separation of the dense outer portion of the bone (the cortex) from the less dense trabecular bone.

The vertebra was imported into Fiji and the region of interest was drawn to include the trabecular space in the vertebral body by using the polygon tool with interpolation using the ROI manager. A mask was created from the ROI and imported into Definiens Developer XD 2.1.1 together with the original scan. The region of interest was reduced to 5 pixels $(50 \mu \mathrm{m})$ in all directions to ensure that no parts of the cortical bone were included and segmented using a 
threshold of $700 \mathrm{mg} \mathrm{HA} / \mathrm{cm}^{3}$. The segmentation was exported as image stacks using black for enclosed void, white for bone and indicating outside of the region of interest. The segmented images were then imported in Fiji and measured using the BoneJ plugin.

\subsection{Statistical Analysis}

Statistical differences in bone microstructure, bone microRNA/mRNA transcript levels, and serum microRNA transcript levels between SHAM and the three OVX groups were conducted using GraphPad Prims v5.03. Statistical significance of group differences was assessed using 1-way-ANOVA in conjunction with Tukey post hoc tests for pair-wise comparisons. Scatterplots present the distribution of values as well as mean +/- standard deviation. Statistical analysis of small RNA sequencing data based on TPM values was performed using the methods available under the EdgeR package in R/Bioconductor. P-value obtained from EdgeR were adjusted for multiple testing using Benjamini-Hochbergs false discovery rates (FDR) ${ }^{(28)}$. 


\section{RESULTS}

\subsection{Study aim and experiment design}

The aim of the TAMIBAT study is the systematic investigation of microRNA levels in rodent bone tissue and cell-free blood (serum) during the progression of hormone-induced bone loss and in response to anabolic and anti-resorptive rescue of bone loss, respectively. Figure 1 summarizes the experiment's design: 42 six-month-old Sprague Dawley rats were included into the study and randomized to OVX or SHAM treatment. Eight weeks after surgery, OVX animals were randomized to treatment with vehicle solution (VEH), teriparatide (TPD), or zoledronate (ZOL). Treatments as well as multiple blood withdrawals and in vivo micro-CT analysis were performed within a time frame of 12 weeks, ending with sacrifice of all animals and collection of blood and bone tissue for gene expression and ex vivo nano-CT analysis (Fig. 1A). Initially, we were interested in the effects of bone loss as well as therapy on bone tissue microRNA levels. Therefore, we applied next-generation sequencing (NGS) for the discovery of changes in small RNAs, specifically miRNAs, on tissue level and RT-qPCR to confirm these findings in the complete sample set (Fig. 1B).

\subsection{Bone Microarchitecture}

We observed a significant reduction in trabecular bone at week 20 in ovariectomized and untreated animals (VEH) at sites of tibial and vertebral bone (Fig 2). Anabolic treatment (TPD) resulted in a complete rescue of bone loss after 20 weeks, while anti-resorptive treatment (ZOL) resulted in only a partial rescue of BV/TV. These effects were similarly observed at both the tibia and vertebral bone sites. 


\subsection{MicroRNA expression in femoral head in response to anti-osteoporosis therapy}

To investigate changes in bone microRNA expression as a consequence of ovariectomy $(\mathrm{OVX})$ and in response to treatment, femoral head samples were harvested at week 20 and used for RNA extraction. Initially, NGS was used for a genome-wide screen of miRNA levels in a subset of 15 out of 42 samples (6x SHAM, 3x VEH, 3x TPD, 3x ZOL). Samples were selected based on nano-CT results. NGS detected 426 distinct miRNAs in femoral head tissue, which yielded at least one tag per million (TPM) in at least one out of the 15 samples. Out of the 426 detected miRNAs, 273 gave one or more TPMs in each of the 15 samples and were subsequently used for statistical analysis. Using TPMs from the most variant microRNAs (top 30 sorted by CV\%) a heatmap was generated (Supporting Figure 2), which showed distinct microRNA signatures in vehicle treated OVX animals that was clearly different to that of SHAM, TPD and ZOL animals. In contrast, miRNA expression in bone of SHAM and treated animals was observed to be more similar. Next, we performed statistical analysis of the group differences using EdgeR. P-values were adjusted for multiple testing and an adjusted p-value cut-off of $0.1(\mathrm{FDR}=10 \%)$ was applied to identify differentially expressed miRNAs. Comparing vehicle-treated OVX animals (VEH) to SHAM controls we identified 13 significantly up-regulated miRNAs in VEH compared to SHAM control, while no miRNA was down-regulated (Fig. 3A). Anabolic treatment using teriparatide had profound effects on bone miRNA expression compared to vehicle-treated controls (VEH) and resulted in a significant regulation of 46 miRNAs (21 up, 25 down) (Fig. 3B), while 10 miRNAs (1 up, 9 down) were found to be regulated between ZOL and VEH (Figure 3C). Seven miRNAs (miR-203a-3p, miR-17-5p, miR-335-5p, miR-132-3p, miR-34a-5p, miR-19a-3p, miR-181b$3 p)$ which showed significant differential expression in any of these comparisons were successfully replicated by RT-qPCR to confirm the validity of NGS results (Supporting Figure 3). 


\section{4.miR-203a expression is up-regulated in bone of ovariectomized animals and}

\section{rescued by treatment}

In the NGS screen miR-203a/b-3p showed the highest up-regulation in bone of OVX animals receiving vehicle $(\mathrm{VEH})$ treatment compared to SHAM controls $\left(\log _{2} \mathrm{FC}=1.5, \mathrm{FDR}<0.001\right.$, see Fig. 3A). Conversely, anabolic treatment with teriparatide led to a significant downregulation of miR-203a in femoral head compared to vehicle-treated animals $\left(\log _{2} \mathrm{FC}=-0.85\right.$, FDR $=0.045$, see Fig. 3B). Similarly, zoledronate $(\mathrm{ZOL})$ treated femoral head bone tissue exhibited lower miR-203b expression $\left(\log _{2} \mathrm{FC}=-1.17\right.$, FDR $\left.=0.04\right)$. Accordingly, we selected miR-203a for further investigation.

Levels of miR-203a were first analyzed in the entire cohort of 42 animals by RT-qPCR to confirm NGS results and increase sample size. Up-regulation of miR-203a in VEH versus SHAM group and rescue due to teriparatide treatment were confirmed $(\mathrm{p}<0.05$, Fig. 4A). Although treatment with zoledronic acid did not result in a rescue of miR-203a levels, levels were lower by trend compared to VEH animals (Fig. 4A). RT-qPCR was used to quantify mRNA levels of two transcription factors that are regulated by miR-203a, namely Dlx 5 and Runx2, as well as Osterix (Osx), which acts down-stream of Runx2. The regulation of D1x5 was inverse to miR-203a, indicated by down-regulation in the OVX group with vehicle treatment $(\mathrm{VEH})$ and up-regulation upon teriparatide treatment $(\mathrm{TPD})(\mathrm{p}<0.05$, Fig. 4B). Likewise, Runx2 was observed to be down-regulated in bone of untreated OVX animals (VEH) compared to SHAM (Fig 4C). However, treatment with teriparatide did not result in an induction of Runx 2 mRNA levels. Osterix mRNA levels showed the same trend as Runx2 $(\mathrm{r}=0.84, \mathrm{p}<0.001)$ but in contrast to Runx2, the down-regulation in VEH vs SHAM did not reach significance. 


\subsection{Serum levels of miR-203a are significantly correlated to expression in bone}

In order to monitor circulating levels of miR-203a after 12 weeks of treatment, total RNA was extracted from serum samples of all 42 animals included in the TAMIBAT study at week 20. We observed a significant correlation $(r=0.41, p<0.05)$ between miR-203a-3p levels in bone and matched serum samples (Fig. 5).

\section{DISCUSSION}

In this classical animal model for postmenopausal osteoporosis, the expression of bonerelated miRNAs was investigated under teriparatide and zoledronic acid treatment, respectively. Although hormone replacement therapy (HRT) shows advantageous effects on bone metabolism, it is not considered first line therapy for the treatment of osteoporosis. Therefore, the aim of the present study was to investigate the effects of an established antiresorptive or an osteo-anabolic agent, as the mode of action as well as the effects on bone mineral density and fracture risk reduction are different ${ }^{(29)}$.

Total RNA was extracted from femoral head tissue, as this bone can be collected quickly and reproducibly, thus enabling the ability to obtain high quality RNA from bone cells and to a lesser extent bone marrow cells. Circulating miRNAs were analyzed using cell-free blood (serum) of ovariectomized rats and SHAM controls. Ovariectomy without therapy, reflecting postmenopausal osteoporosis, as well as anti-resorptive and osteo-anabolic treatment resulted in distinct miRNA changes in bone tissue. In total, 11 miRNAs were up-regulated in untreated OVX animals and down-regulated under a teriparatide or zoledronate treatment regimen. These miRNAs seem be mechanistically relevant regarding postmenopausal bone loss and treatment response. 
miR-203a showed the most significant up-regulation in bone of ovariectomized untreated rats compared to SHAM, which was rescued by teriparatide treatment. miR-203a was previously described to be an important regulator of bone formation, as it is involved in the differentiation of mesenchymal stem cells. For example, down-regulation of miR-203a promotes osteoblastic differentiation by up-regulation of osteogenic effectors including PPAP2B, NEGR1 and PDGFRA (30). In addition, miR-203a acts as a potent inhibitor of Dlx5, which regulates the transcription factors Osx and Runx2. A direct interaction between miR203a and Runx2 has been proposed in a breast cancer model (31). Consequently, osteoblast differentiation is slowed by miR-203a (32). In line with these previous observations, elevated expression of miR-203a was found in the present study in femoral head tissue and serum of vehicle-treated OVX rats, representing the untreated postmenopausal osteoporotic phenotype, suggesting that miR-203a might contribute to delayed osteogenic differentiation and hence development of bone loss. Contrarily, inhibition of miR-203a in vitro resulted in the stimulation of matrix mineralization in the study of Laxman et al. (32). Laxman et al. have shown that the transcription of miR-203a itself seems to be negatively controlled by BMP2,

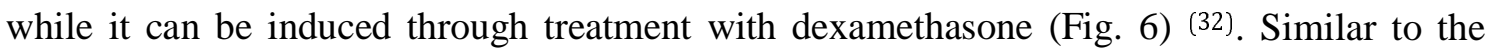
previously described up-regulation of miR-203a in cell-free blood of untreated OVX rats, miR-203a was also found to be up-regulated in serum of diabetic and non-diabetic postmenopausal women with osteoporosis and a history of low-traumatic fractures (17). Taken together, these findings suggest an important role for miR-203a in the regulation of bone formation and potentially during the onset and development of postmenopausal osteoporosis. In bone samples of untreated ovariectomized rats we observed that both Dlx 5 and Runx 2 were down-regulated in comparison to SHAM animals. However, low Runx2 levels were also found in the teriparatide (TPD) treated group. These findings are somewhat surprising as TPD is an anabolic agent and bone microstructure and density increased significantly following 12weeks of treatment, suggesting a sufficient treatment response. The increased transcription of 
Runx2 during bone formation is believed to be oscillating rather than constant, indicating that the time point of analysis may be essential to detect up-regulation. In addition, mRNA and protein levels are not necessarily correlated, especially due to the action of posttranscriptional regulators such as miRNAs. For example, a study on TPD-treated osteoblastic MC3T3-E1 cells observed an unexpected down-regulation of Runx2 as well. In this study, femoral bone tissue was taken from rats 48 hours after the last treatment with TPD, which might be responsible for the observed effect. It has to be taken into account, that the mechanisms of osteo-anabolic drugs are complex. In addition to Runx 2 other mechanisms including the Wnt/ $\beta$-catenin pathway are responsible for osteoblast differentiation under TPDtreatment ${ }^{(33)}$. miR-203a might be an important regulator of osteoblast differentiation. However, it does not reflect the whole body microenvironment.

Another key observation made by this study is that miR-203a levels in bone tissue and serum are significantly regulated. This finding confirms that changes in miRNA levels in bone tissue can be detected using liquid biopsies. A similar concomitant up- or down-regulation of miRNAs in bone tissue, serum as well as in osteoblasts and osteoclasts was also reported by other study groups $(34,35)$ and encourages the potential clinical utility of miRNAs as bone biomarkers.

Apart from miR-203a, miR-20a-5p levels were also up-regulated following ovariectomy and down-regulated due to TPP therapy. miR-20a-5p was recently reported to be one of the most abundant miRNAs in bone tissue from postmenopausal women (36). Similar to miR-203a, miR-20a-5p also regulates the activity of transcription factors of osteoblast differentiation such as Runx2, BMP2 and PPAR $\gamma(36,37)$ and thereby osteoblast differentiation and bone formation. 
Currently no other data on miRNAs in models of bisphosphonate treated postmenopausal osteoporosis are available. Teriparatide and denosumab therapy changed circulating miRNAs in postmenopausal osteoporosis in the study of Anastasilakis et al. (22). In this study, miRNA133 was down-regulated under teriparatide, but not denosumab therapy, suggesting an inhibitory role of miRNA-133 on the RUNX-2 gene and therefore osteoblastogenesis ${ }^{(22) . ~ I n ~}$ the present study, miRNA-133 was down-regulated in animals receiving zoledronic acid, suggesting a positive effect of bisphosphonates on RUNX2 and thus bone formation. Significantly different expressions of miRNAs, including bone-related miR-29b-3p, were found between estrogenic hormone replacement therapy users and their treatment-naïve twins (38). If estrogen replacement can rescue or reverse the effects of estrogen deficiency on miRNAs is an important issue and should be raised in further research projects.

\section{CONCLUSION}

By taking advantage of a standardized animal model of postmenopausal osteoporosis, profound effects of hormone-induced bone loss as well as osteo-anabolic and anti-resorptive treatment on miRNA expression in bone, specifically femoral head, were revealed. Therapeutic intervention, especially osteo-anabolic therapy, resulted in a restoration of miRNA expression similar to sham-operated animals. Among the affected miRNAs, the induction of miR-203a in bone of ovariectomized rats was most significant and, notably, also detectable in cell-free blood. The regulation of miR-203a also affected mRNA levels of its direct targets, the transcription factors Dlx5 and Runx2, in femoral head tissues. 


\section{ACKNOWLEDGEMENT}

The authors acknowledge T.A. Vacca from Linz/Austria for proofreading.

\section{CONTRIBUTION}

Individual contributions include the following: R.K., M.H., P.P., J.G. and H.R. for the study concept and design; M.W., E.G. and S.S. for the data collection; P.H. and R.K. for $\mu$ CT imaging; M.H., M.W., S.S. and E.G. for miRNA analysis; G.L. and J.F. for the animal care; R.K., M.H. and M.W. for the data analysis; R.K., M.H., P.P., H.R. and J.G. for the data interpretation; R.K., M.H. and M.W. for drafting the manuscript and literature research;

J.G., H.R. and P.P. for the extensive revision of the manuscript. 


\section{REFERENCES}

1. Nanes MS, Kallen CB. Osteoporosis. Seminars in Nuclear Medicine. 2014 Nov;44(6):439-50.

2. Maeda SS, Lazaretti-Castro M. An overview on the treatment of postmenopausal osteoporosis. Arquivos brasileiros de endocrinologia e metabologia. 2014 Mar;58(2):162-71.

3. Odén A, McCloskey E V., Kanis JA, Harvey NC, Johansson H. Burden of high fracture probability worldwide: secular increases 2010-2040. Osteoporosis International. Springer London; 2015 Sep 28;26(9):2243-8.

4. Garnero P. The Utility of Biomarkers in Osteoporosis Management. Molecular Diagnosis \& Therapy. 2017 Aug 7;21(4):401-18.

5. Eastell R, Szulc P. Use of bone turnover markers in postmenopausal osteoporosis. The lancet. Diabetes \& endocrinology. Elsevier; 2017 Nov 1;5(11):908-23.

6. Hackl M, Heilmeier U, Weilner S, Grillari J. Circulating microRNAs as novel biomarkers for bone diseases - Complex signatures for multifactorial diseases? Molecular and Cellular Endocrinology. 2016;

7. Sera SR, Zur Nieden NI. microRNA Regulation of Skeletal Development. Current osteoporosis reports. NIH Public Access; 2017;15(4):353-66.

8. Smith-Vikos T, Slack FJ. MicroRNAs and their roles in aging. Journal of cell science. Company of Biologists; 2012 Jan 1;125(Pt 1):7-17.

9. Zhao X, Xu D, Li Y, Zhang J, Liu T, Ji Y, Wang J, Zhou G, Xie X. MicroRNAs regulate bone metabolism. Journal of Bone and Mineral Metabolism. Springer Japan; 2014 May 6;32(3):221-31.

10. Shenoy A, Blelloch RH. Regulation of microRNA function in somatic stem cell proliferation and differentiation. Nature reviews. Molecular cell biology. NIH Public Access; 2014 Sep;15(9):565-76.

11. Taipaleenmäki H. Regulation of Bone Metabolism by microRNAs. Current Osteoporosis Reports. 2018;16(1).

12. Cheng VK-F, Au PC-M, Tan KC, Cheung C-L. MicroRNA and Human Bone Health. JBMR Plus. 2018;3(1):2-13.

13. Riikka Mäkitie, Hackl M, Niinimäki R, Kakko S, Grillari J, Mäkitie O. Altered MicroRNA Profile in Osteoporosis Caused by Impaired WNT Signaling. JCEM. 2018;

14. Fan X, Bi X, Wu S, Gu P, Hu Y, Deng Y, Zhou H, Wang Y. Effects of a miR-31, Runx2 , and Satb2 Regulatory Loop on the Osteogenic Differentiation of Bone Mesenchymal Stem Cells. Stem Cells and Development. 2013;22(16):2278-2286.

15. Deng Y, Bi X, Zhou H, You Z, Wang Y, Gu P, Fan X. Repair of critical-sized bone defects with anti-miR-31-expressing bone marrow stromal stem cells and poly(glycerol sebacate) scaffolds. European Cells and Materials. 2014;27:13-25. 
16. An JH, Ohn JH, Song JA, Yang J-Y, Park H, Choi HJ, Kim SW, Kim SY, Park W-Y, Shin CS. Changes of MicroRNA Profile and MicroRNA-mRNA Regulatory Network in Bones of Ovariectomized Mice: MICRORNA PROFILE CHANGES BY OVARIECTOMY. Journal of Bone and Mineral Research. 2014 Mar;29(3):644-56.

17. Heilmeier U, Hackl M, Skalicky S, Weilner S, Schroeder F, Vierlinger K, Patsch JM, Baum T, Oberbauer E, Lobach I, Burghardt AJ, Schwartz A V, Grillari J, Link TM. Serum miRNA Signatures Are Indicative of Skeletal Fractures in Postmenopausal Women With and Without Type 2 Diabetes and Influence Osteogenic and Adipogenic Differentiation of Adipose Tissue-Derived Mesenchymal Stem Cells In Vitro. Journal of Bone and Mineral Research Month. 2016;xx(xx):1-20.

18. Yavropoulou MP, Anastasilakis AD, Makras P, Tsalikakis DG, Grammatiki M, Yovos JG. Expression of microRNAs that regulate bone turnover in the serum 1 of postmenopausal 2 women with low bone mass and vertebral fractures. European Journal of Endocrinology. 2017;176(2):169-76.

19. Ding H, Meng J, Zhang W, Li Z, Li W, Zhang M, Fan Y, Wang Q, Zhang Y, Jiang L, Zhu W. Medical examination powers miR-194-5p as a biomarker for postmenopausal osteoporosis. Scientific Reports. Springer US; 2017;7(1):1-11.

20. Feichtinger X, Muschitz C, Heimel P, Baierl A, Fahrleitner-Pammer A, Redl H, Resch H, Geiger E, Skalicky S, Dormann R, Plachel F, Pietschmann P, Grillari J, Hackl M, Kocijan R. Bone-related Circulating MicroRNAs miR-29b-3p, miR-550a-3p, and miR324-3p and their Association to Bone Microstructure and Histomorphometry. Scientific Reports. 2018;8(1):1-11.

21. Kocijan R, Muschitz C, Geiger E, Skalicky S, Baierl A, Dormann R, Plachel F, Feichtinger X, Heimel P, Fahrleitner-Pammer A, Grillari J, Redl H, Resch H, Hackl M. Circulating microRNA signatures in patients with idiopathic and postmenopausal osteoporosis and fragility fractures. JCEM. 2016;

22. Anastasilakis AD, Makras P, Pikilidou M, Tournis S, Makris K, Bisbinas I, Tsave O, Yovos JG, Yavropoulou MP. Changes of Circulating MicroRNAs in Response to Treatment With Teriparatide or Denosumab in Postmenopausal Osteoporosis. The Journal of Clinical Endocrinology \& Metabolism. 2018 Mar 1;103(3):1206-13.

23. Gasser JA, Ingold P, Venturiere A, Shen V, Green JR. Long-term protective effects of zoledronic acid on cancellous and cortical bone in the ovariectomized rat. Journal of Bone and Mineral Research. 2008;23(4):544-51.

24. Brouwers JEM, Lambers FM, Gasser JA, van Rietbergen B, Huiskes R. Bone degeneration and recovery after early and late bisphosphonate treatment of ovariectomized wistar rats assessed by in vivo micro-computed tomography. Calcified Tissue International. 2008;82(3):202-11.

25. Blondal T, Jensby Nielsen S, Baker A, Andreasen D, Mouritzen P, Wrang Teilum M, Dahlsveen IK. Assessing sample and miRNA profile quality in serum and plasma or other biofluids. Methods. Elsevier Inc.; 2013;59(1):164-9.

26. Carter LE, Kilroy G, Gimble JM, Floyd ZE. An improved method for isolation of RNA from bone. BMC Biotechnology. 2012 Jan 19;12(1):5. 
27. Metsalu T, Vilo J. ClustVis: a web tool for visualizing clustering of multivariate data using Principal Component Analysis and heatmap. Nucleic acids research. 2015 Jul 1;43(W1):W566-70.

28. Robinson MD, McCarthy DJ, Smyth GK. edgeR: a Bioconductor package for differential expression analysis of digital gene expression data. Bioinformatics (Oxford, England). Oxford University Press; 2010 Jan 1;26(1):139-40.

29. Kendler DL1, Marin F2, Zerbini CAF3, Russo LA4, Greenspan SL5, Zikan V6, Bagur A7, Malouf-Sierra J8, Lakatos P9, Fahrleitner-Pammer A10, Lespessailles E11, Minisola S12, Body JJ13, Geusens P14, Möricke R15, López-Romero P2. Effects of teriparatide and risedronate on new fractures in post-menopausal women with severe osteoporosis (VERO): a multicentre, double-blind, double-dummy, randomised controlled trial. Lancet. 2018 Jan 20;391(10117):230-240.

30. Xu X, Jiang H, Li X, Wu P, Liu J, Wang T, Zhou X, Xiong J, Li W. Bioinformatics analysis on the differentiation of bone mesenchymal stem cells into osteoblasts and adipocytes. Molecular medicine reports. 2017 Apr;15(4):1571-6.

31. Taipaleenmäki H, Browne G, Akech J, Zustin J, van Wijnen AJ, Stein JL, Hesse E, Stein GS, Lian JB. Targeting of Runx2 by miR-135 and miR-203 Impairs Progression of Breast Cancer and Metastatic Bone Disease. Cancer research. NIH Public Access; 2015 Apr 1;75(7):1433-44.

32. Laxman N, Mallmin H, Nilsson O, Kindmark A. miR-203 and miR-320 Regulate Bone Morphogenetic Protein-2-Induced Osteoblast Differentiation by Targeting Distal-Less Homeobox 5 (Dlx5). Genes. Multidisciplinary Digital Publishing Institute (MDPI); 2016 Dec 23;8(1).

33. Tian Y, Xu Y, Fu Q, He M. Parathyroid hormone regulates osteoblast differentiation in a Wnt/ $\beta$-catenin-dependent manner. Molecular and cellular biochemistry. 2011 Sep $1 ; 355(1-2): 211-6$.

34. Kelch S, Balmayor ER, Seeliger C, Vester H, Kirschke JS, van Griensven M. miRNAs in bone tissue correlate to bone mineral density and circulating miRNAs are gender independent in osteoporotic patients. Scientific reports. Nature Publishing Group; 2017 Nov 20;7(1):15861.

35. Seeliger C, Karpinski K, Haug AT, Vester H, Schmitt A, Bauer JS, Van Griensven M. Five freely circulating miRNAs and bone tissue miRNAs are associated with osteoporotic fractures. Journal of Bone and Mineral Research. 2014;29(8):1718-28.

36. De-Ugarte L, Serra-Vinardell J, Nonell L, Balcells S, Arnal M, Nogues X, Mellibovsky L, Grinberg D, Diez-Perez A, Garcia-Giralt N. Expression profiling of microRNAs in human bone tissue from postmenopausal women. Human Cell. Springer Japan; 2018 Jan 20;31(1):33-41.

37. Zhang J, Fu W, He M, Xie W, Lv Q, Wan G, Li G, Wang H, Lu G, Hu X, Jiang S, Li J, Lin MCM, Zhang Y, Kung H. MiRNA-20a promotes osteogenic differentiation of human mesenchymal stem cells by co-regulating BMP signaling. RNA Biology. Taylor \& Francis; 2011 Sep 5;8(5):829-38. 
38. Kangas R, Törmäkangas T, Fey V, Pursiheimo J, Miinalainen I, Alen M, Kaprio J, Sipilä S, Säämänen AM, Kovanen V, Laakkonen EK. Aging and serum exomiR content in women-effects of estrogenic hormone replacement therapy. Scientific Reports. 2017;7(January):1-14.

39. Laxman N, Rubin C-J, Mallmin H, Nilsson O, Tellgren-Roth C, Kindmark A. Second generation sequencing of microRNA in Human Bone Cells treated with Parathyroid Hormone or Dexamethasone. Bone. 2016 Mar;84:181-8.

40. Lee MH, Kwon TG, Park HS, Wozney JM, Ryoo HM. BMP-2-induced Osterix expression is mediated by Dlx5 but is independent of Runx2. Biochemical and Biophysical Research Communications. 2003;309(3):689-94. 


\section{FIGURE LEGENDS}

Figure 1. Overview of experiment design and microRNA analysis workflow of the

TAMIBAT study. A) In total 42 Sprague Dawley rats at age 6-months underwent ovariectomy (OVX, n=32) or sham-surgery (SHAM, n=10). After 8 weeks, animals in OVX group were randomized to placebo treatment with vehicle solution $(\mathrm{VEH}, \mathrm{n}=10)$, teriparatide treatment $(\mathrm{TPD}, \mathrm{n}=11)$, or zoledronate treatment $(\mathrm{ZOL}, \mathrm{n}=11)$. Micro-CT, nano-CT, serum and tissue analyses were performed as indicated. B) The microRNA analysis workflow in this study: initially total RNA was extracted from the femoral head of 42 animals. A subset of 15 animals (SHAM, n=6; VEH, $n=3$; TPD, $n=3 ; \mathrm{ZOL}, \mathrm{n}=3$ ) was used for small RNA sequencing. Differentially regulated miRNAs were confirmed by RT-qPCR and targeted analysis of microRNAs in matched serum samples was performed.

Figure 2. Bone microstructure analysis by micro-CT and nano-CT. A/B) Ex vivo nanoCT analysis of the effects on tibial and vertebral bone measured as BV/TV (SHAM, n =10; VEH n=10, TPD, n=11; ZOL n=11). Scatterplots show mean $+/-$ SD. Testing was performed using 1-way ANOVA and Tukey post hoc test for pairwise comparisons. ${ }^{*} \mathrm{p}<0.05, * * \mathrm{p}<0.01$, $* * * \mathrm{p}<0.001$

Figure 3. NGS-discovery of microRNA changes in femoral head bone tissue of ovariectomized animals as well as animals undergoing anti-osteoporotic treatment. A-C) Volcano plots depict the $\log _{2}$ transformed fold change and adjusted p-values for three contrasts (SHAM, $n=6$; VEH, $n=3$; TPD, $n=3$; ZOL, $n=3$ ). microRNA effects with Benjamini-Hochberg adjusted p-values $<0.1$ (FDR $<10 \%$ ) are highlighted as separate group. miR-203a/b is highlighted in red. 
Figure 4. Regulation of miR-203a-3p and confirmed target genes in femoral head bone tissue of the entire TAMIBAT cohort (SHAM, n=10; VEH, n=10; TPD, n=10; ZOL, n=11). A) RT-qPCR quantification of miR-203a. B) RT-qPCR quantification of Dlx5. C) RT-qPCR quantification of Runx2. D) RT-qPCR quantification of Osx. Scatterplots show mean +/- SD. Testing was performed using 1-way ANOVA and Tukey post hoc test for pairwise comparisons. $* \mathrm{p}<0.05, * * \mathrm{p}<0.01, * * * \mathrm{p}<0.001$.

Figure 5. Correlation between bone (x-axis) and serum (y-axis) levels of miR-203a-3p based on $\mathrm{n}=36$ (SHAM, n=9; VEH, n=9; TPD, $\mathrm{n}=8$; ZOL, $\mathrm{n}=10$ ) animals in the TAMIBAT study.

Figure 6. miR-203a is a suppressor of bone formation. PTH, parathyroid hormone; OVX, ovariectomy, DEX, dexamethasone; BMP2, bone morphogenic protein 2. Orange colors indicate the effects that were discovered in this study. References: 1) Laxman N. et al., Bone 2016; (39) 2) Laxman N. et al. Genes (Basel) 2016; (32) 3) Lee M. et al. Biochem Biophys Res Commun.2003; (40) 4) Taipaleenmäki H. Cancer Res. 2015 (31).

\section{Supporting Figure 1. Quality Control of circulating microRNA analysis in rodent serum}

samples. Raw Cq-values obtained for RNA spike-in control (UniSp5) and cDNA spike-in control (cel-miR-39) are plotted for all samples included in the data analysis (SHAM, n=9; VEH, n=9; TPD, n=9; ZOL, n=10). 
Supporting Figure 2. Heatmap. Normalized NGS read counts (TPM-values) for the top 30 microRNAs (ranked according to their coefficient of variation) were used to draw a heatmap. Rows represent microRNAs, columns represent samples. Pearson correlation and complete linkage were used for clustering of samples.

Supporting Figure 3. Confirmation of NGS data by RT-qPCR. In total 15 animal bone tissue samples (SHAM, $\mathrm{n}=6$; VEH, $\mathrm{n}=3$; TPD, $\mathrm{n}=3$; ZOL, $\mathrm{n}=3$ ) were used for NGS analysis. For those samples, the $\log _{2}$-transformed TPM values from the sequencing experiment were plotted against the normalized delta-Cq values obtained from RT-qPCR analysis. 
A

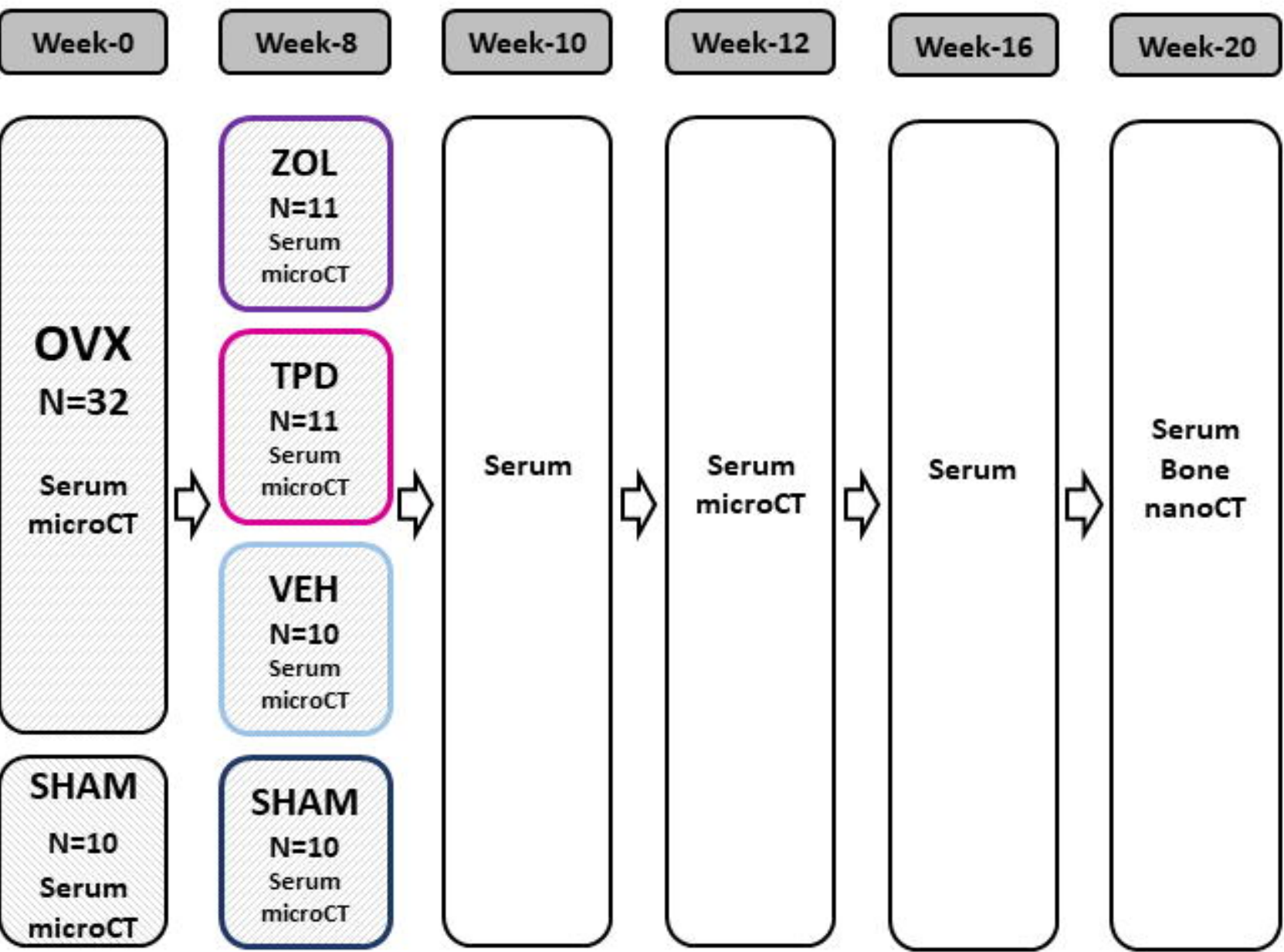

B
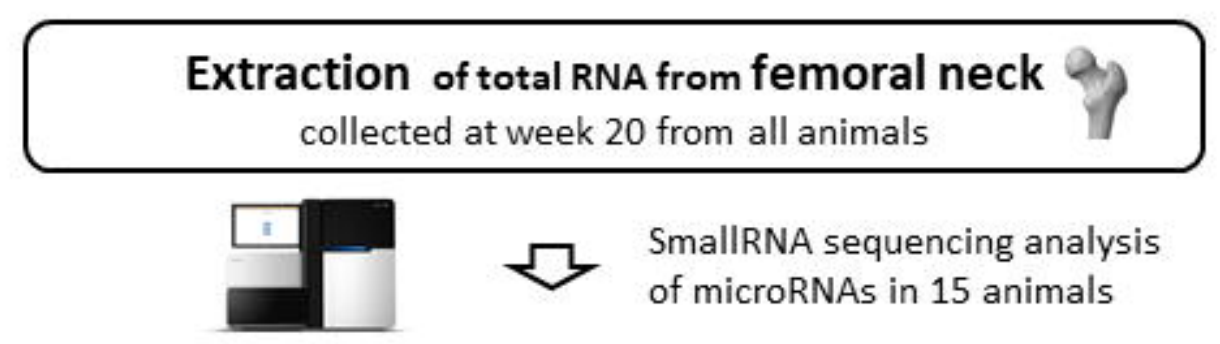

T. SmallRNA sequencing analysis of microRNAs in 15 animals

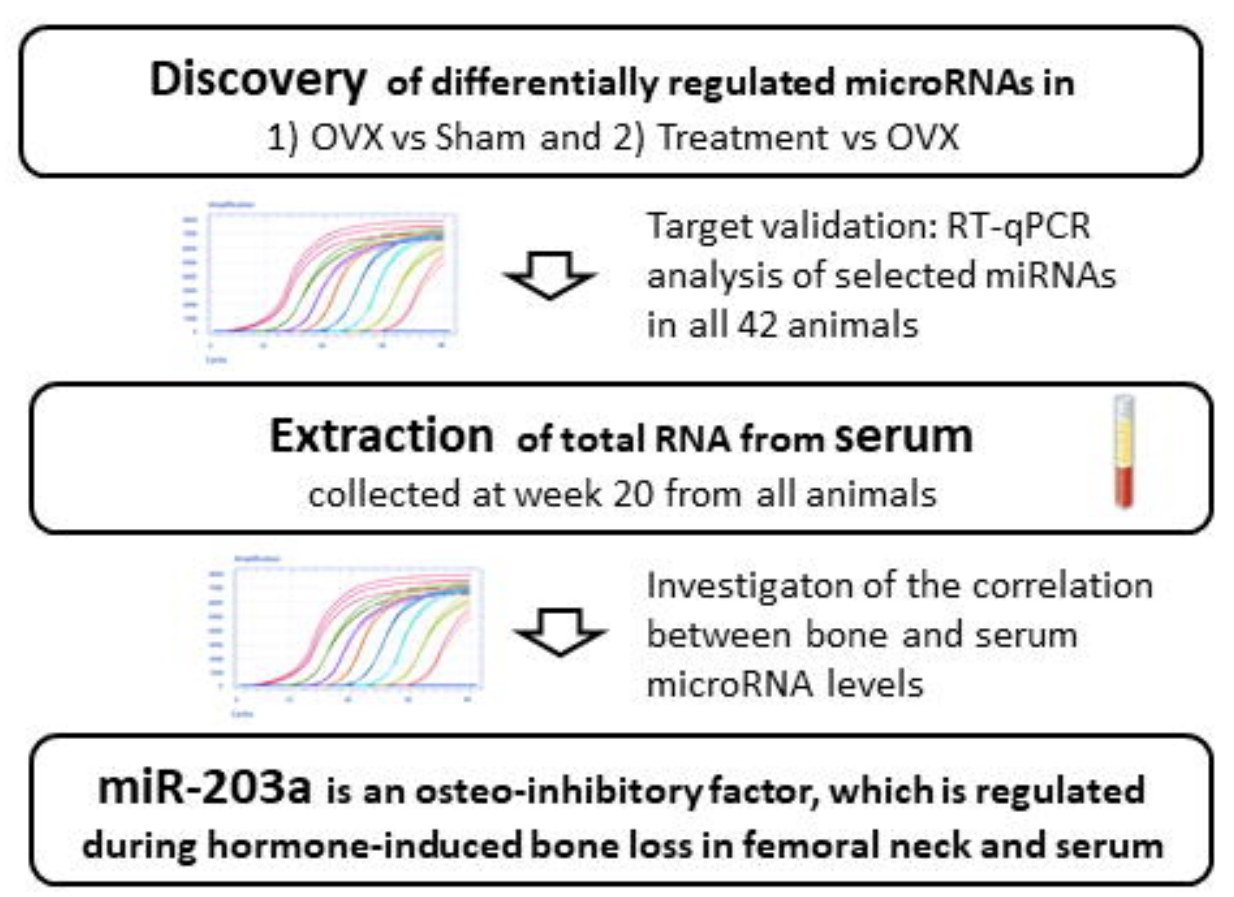


A

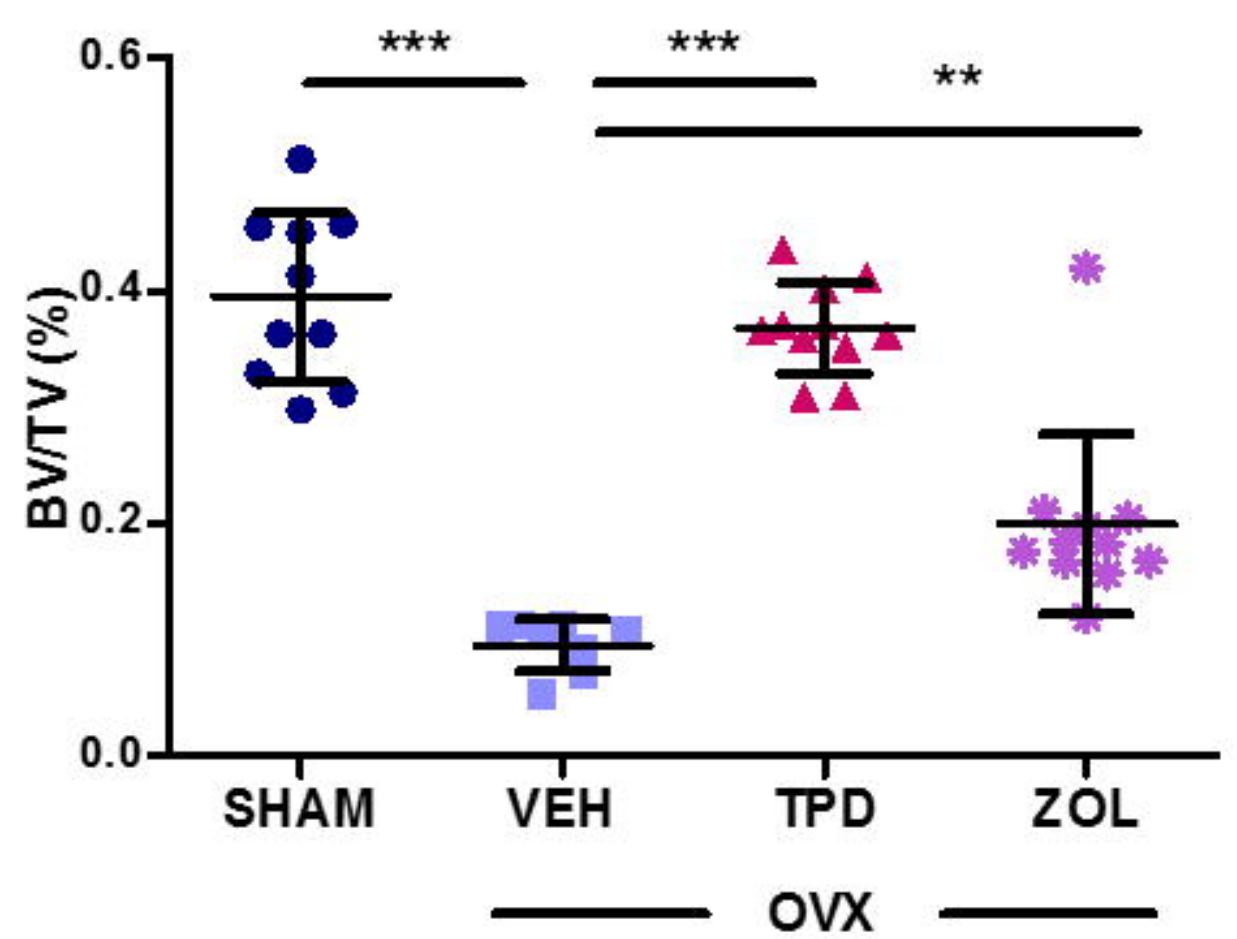

B

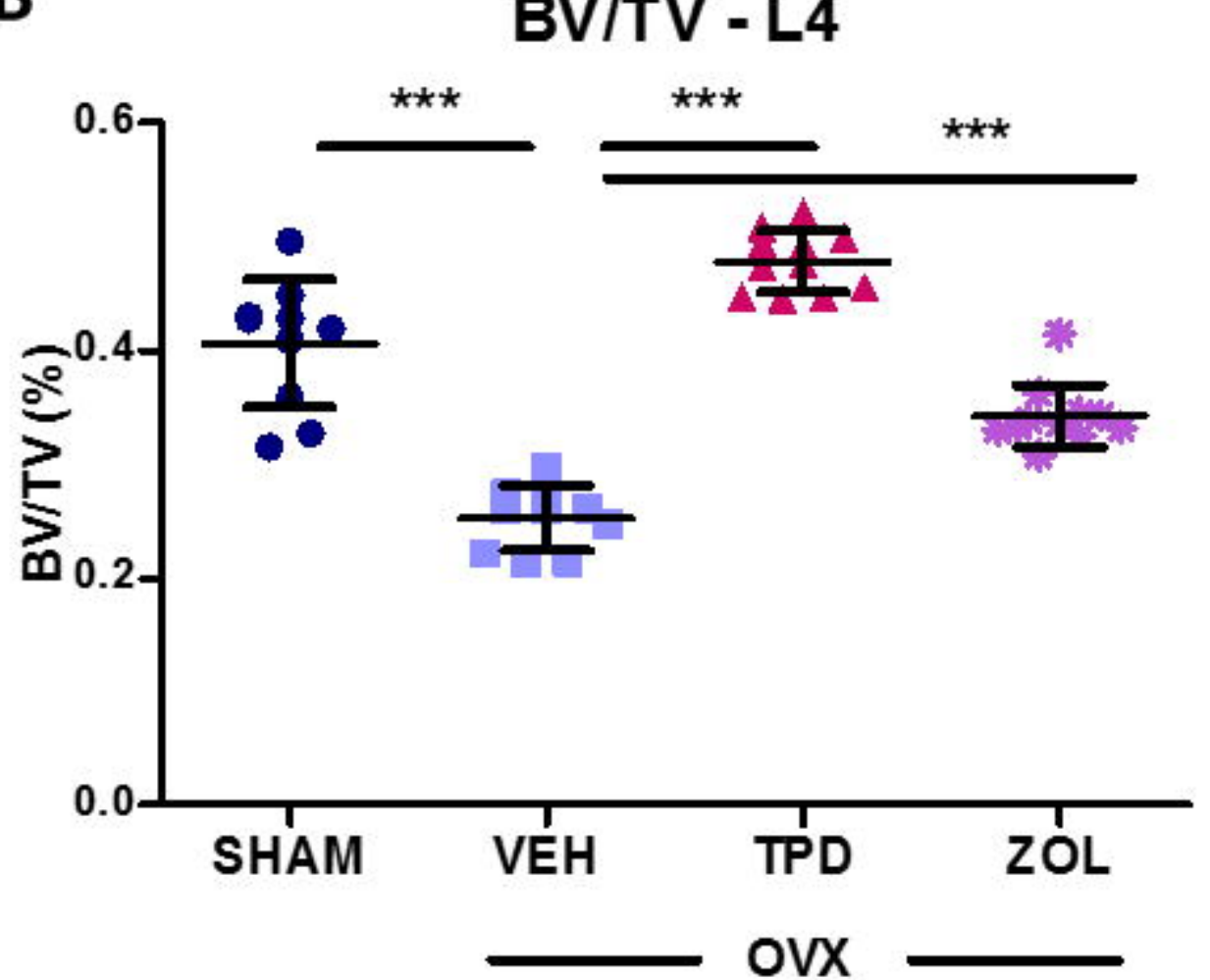




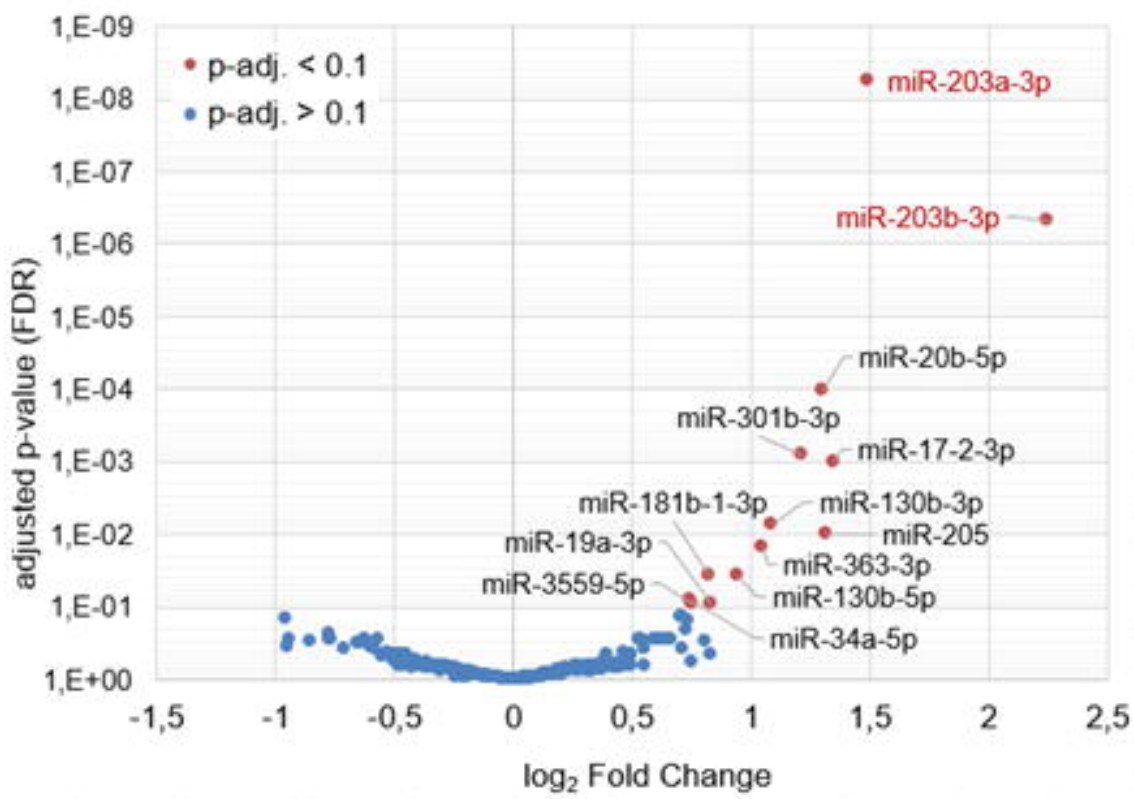

B

TPD $(n=3)$ vs VEH $(n=3)$

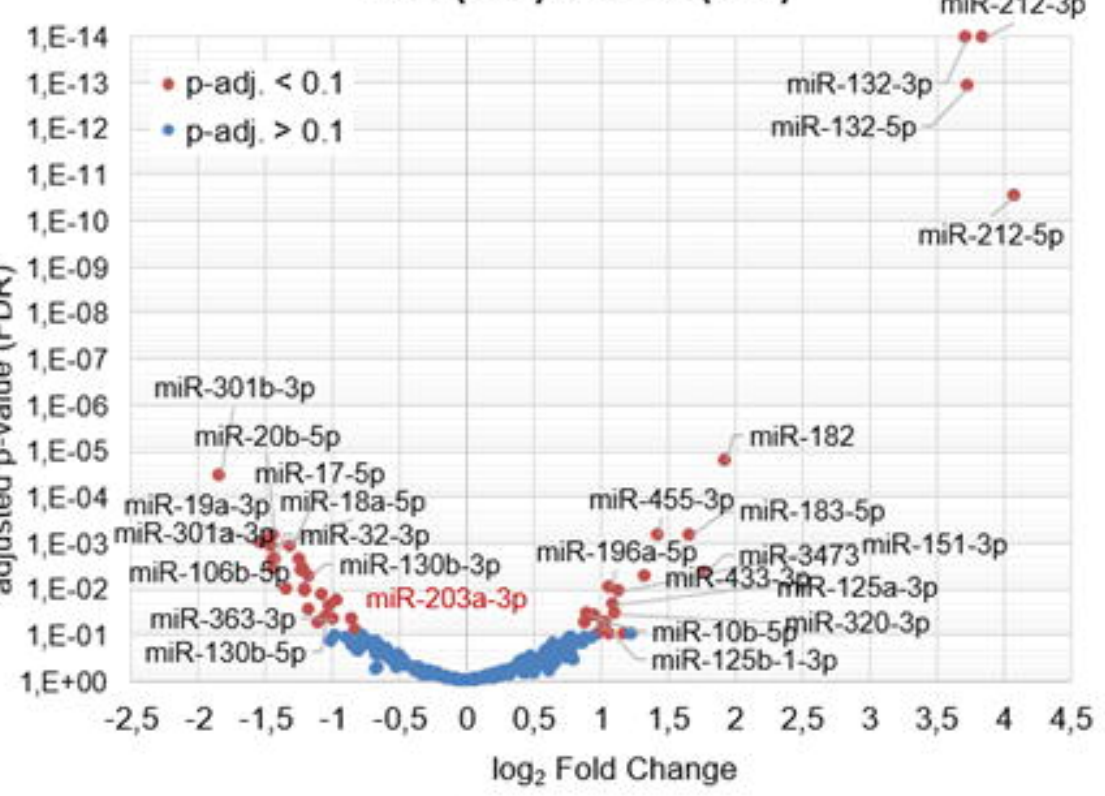

\section{ZOL $(n=3)$ vs VEH $(n=3)$}

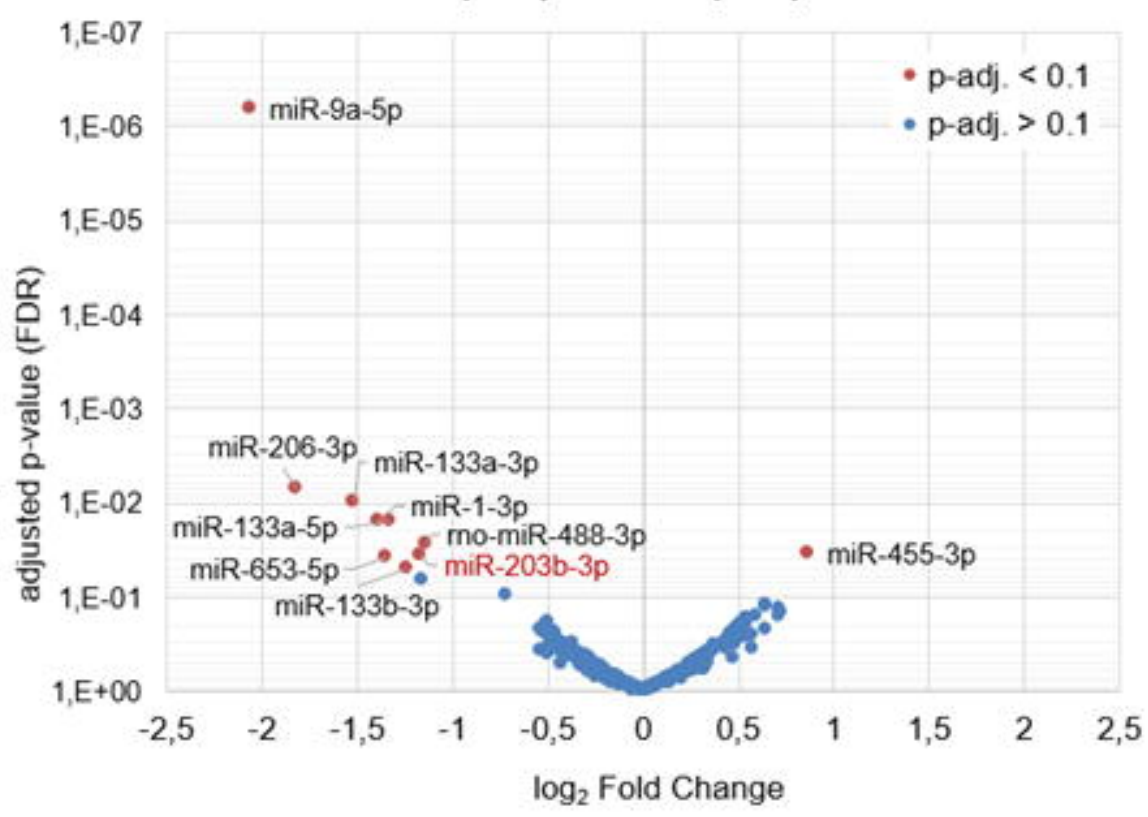


A miR-203a-3p bone expression

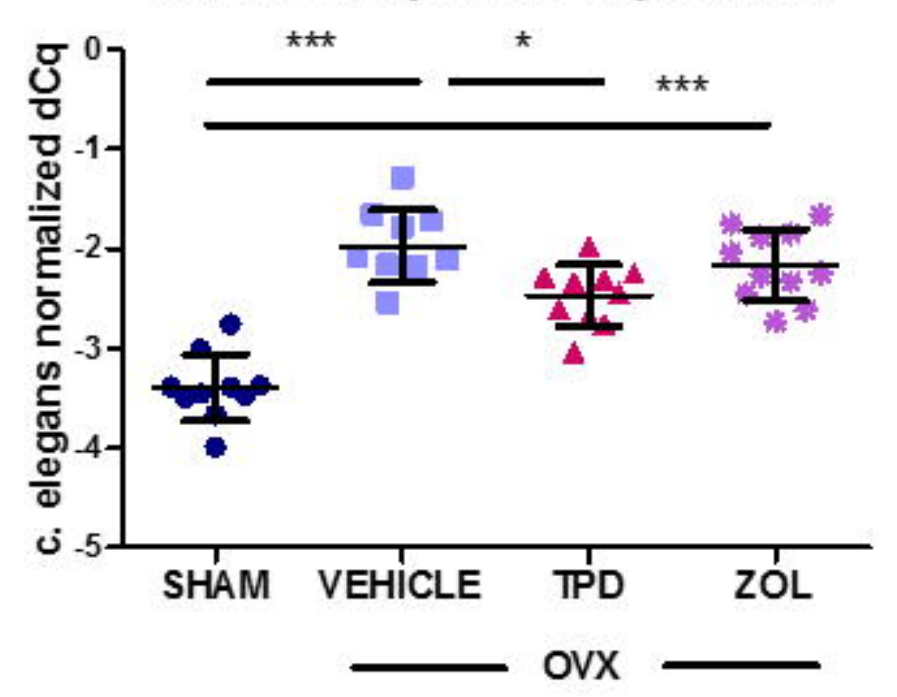

C RUNX2 bone expression

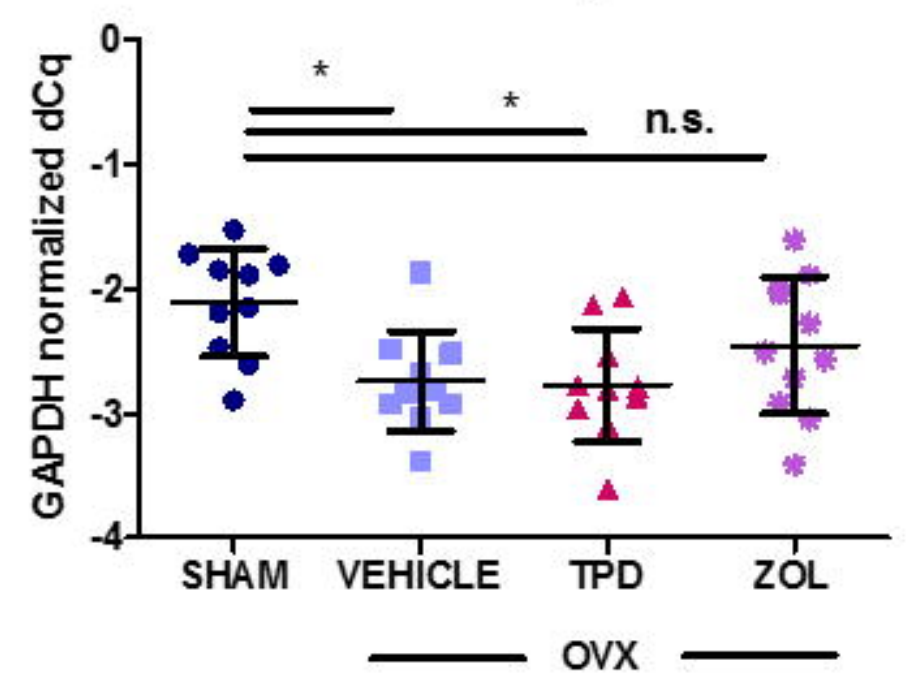

\section{B DIx5 bone expression}

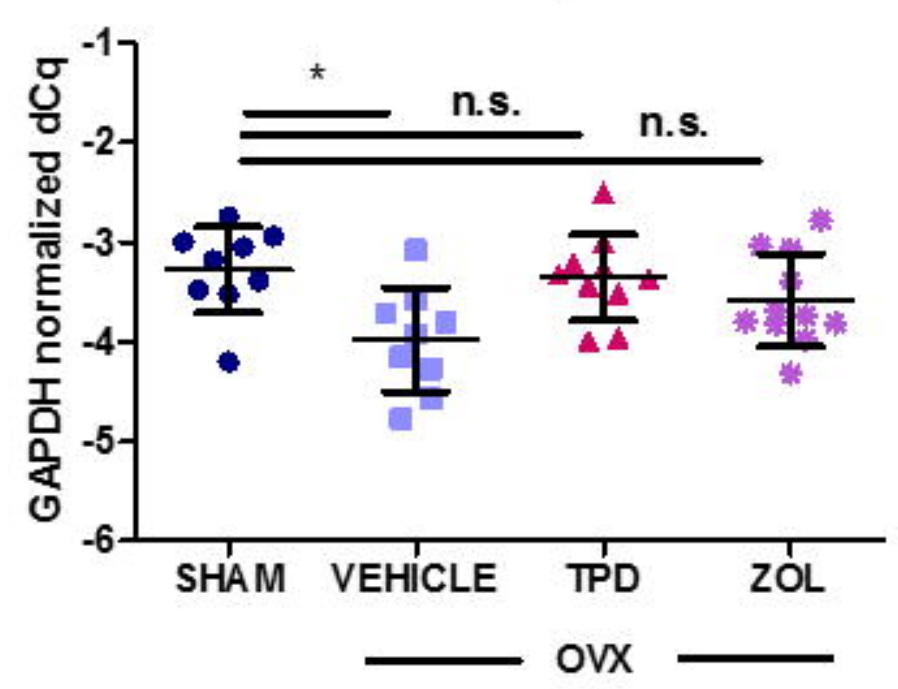

D Osx bone expression

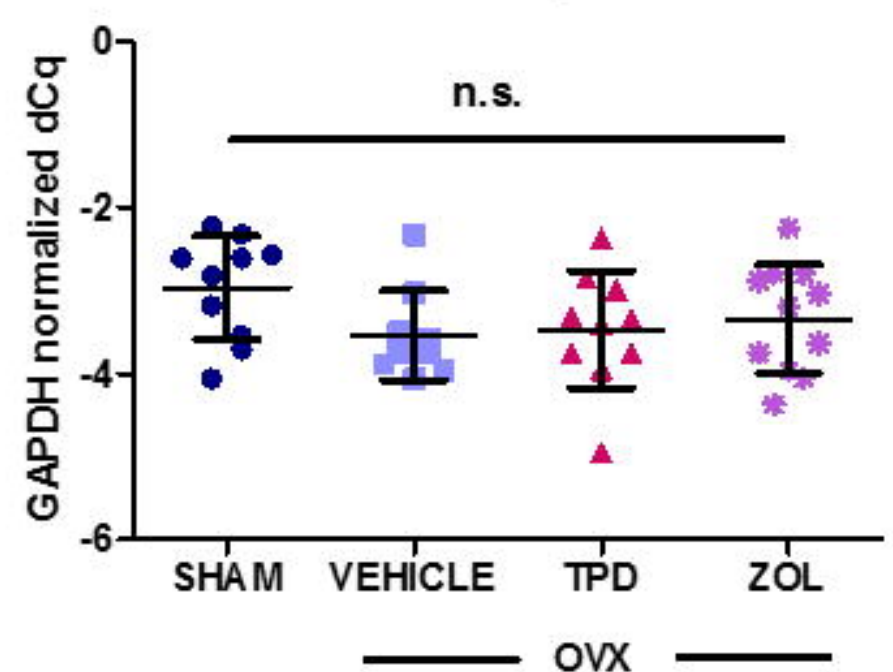




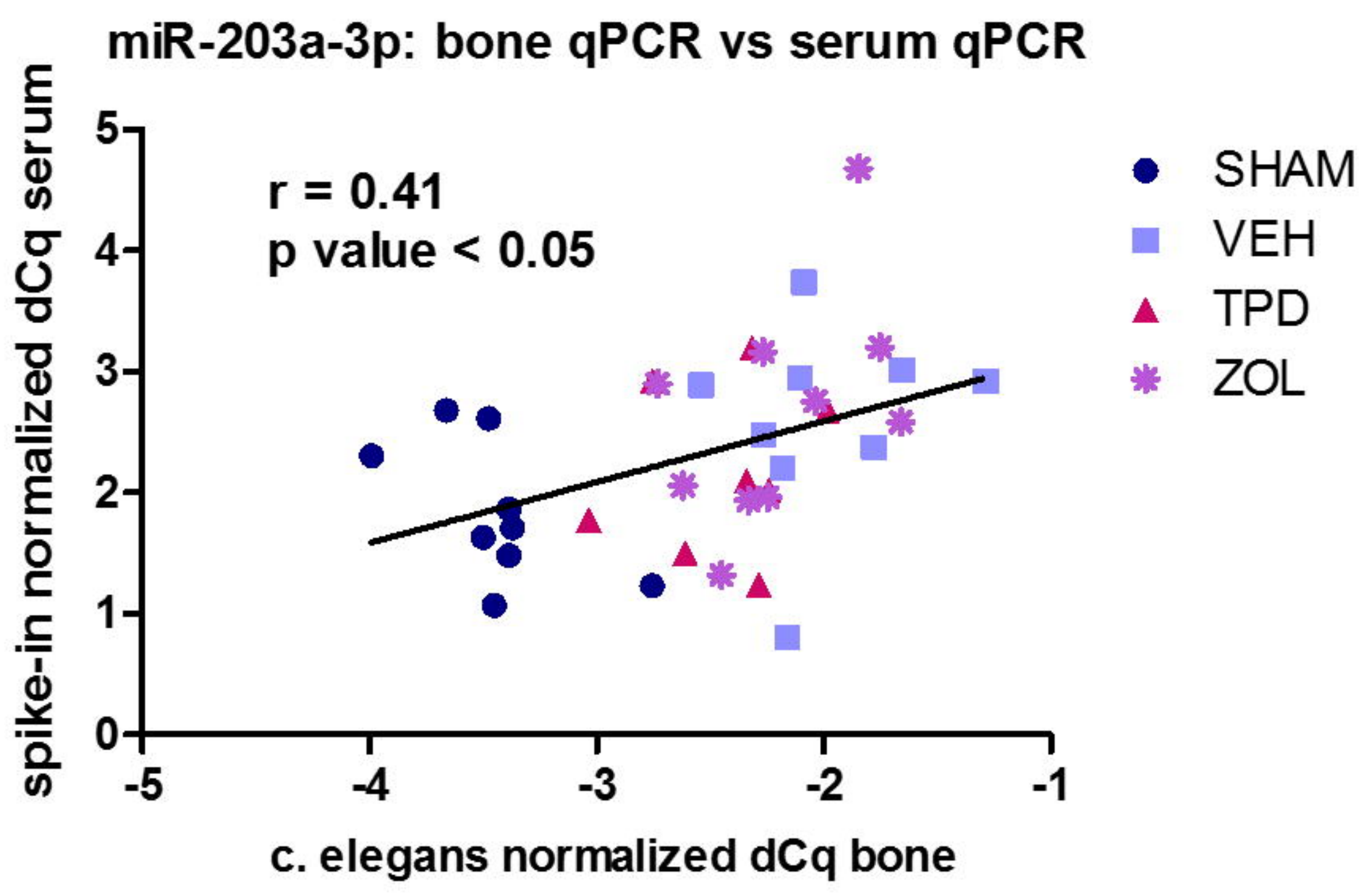




\section{miR-203a is a suppressor of bone formation}

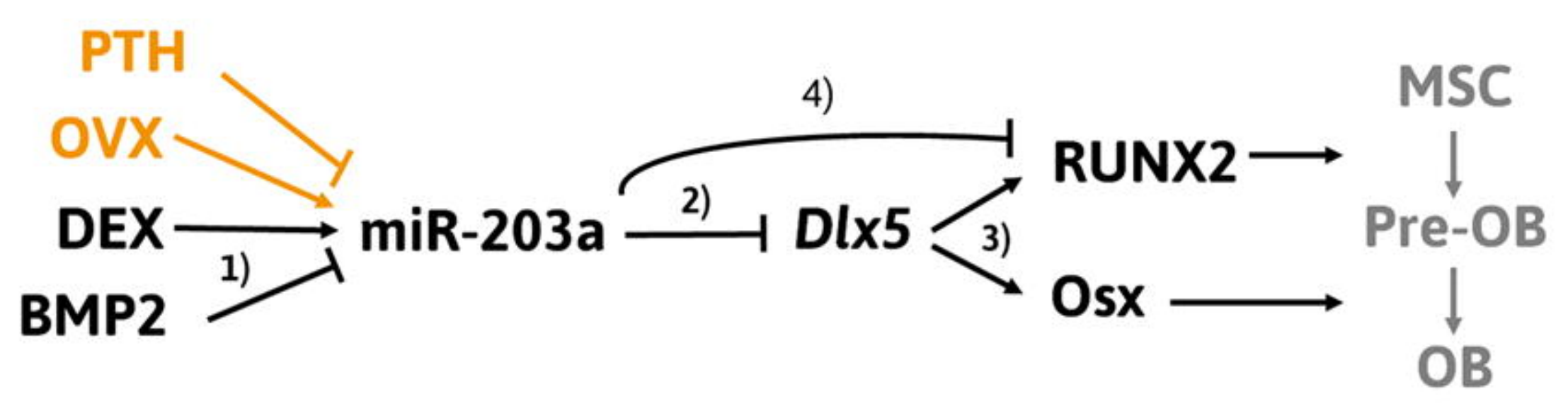

